\title{
Phase field theory of crystal nucleation in hard sphere liquid
}

\author{
László Gránásy, Tamás Pusztai, and Zoltán Jurek \\ Research Institute for Solid State Physics and Optics, H-1525 Budapest, POB 49, Hungary \\ Massimo Conti \\ Dipartimento di Matematica e Fisica, Universita' di Camerino, \\ and Istituto Nazionale di Fisica della Materia, Via Madonna delle Carceri, I-62032, Camerino, Italy \\ Bjørn Kvamme \\ University of Bergen, Department of Physics, Allégaten 55, N-5007 Bergen, Norway
}

(Dated: November 1, 2018)

\begin{abstract}
The phase field theory of crystal nucleation described in [L. Gránásy, T. Börzsönyi, T. Pusztai, Phys. Rev. Lett. 88, 206105 (2002)] is applied for nucleation in hard-sphere liquids. The exact thermodynamics from molecular dynamics is used. The interface thickness for phase field is evaluated from the cross-interfacial variation of the height of the singlet density peaks. The model parameters are fixed in equilibrium so that the free energy and thickness of the (111), (110), and (100) interfaces from molecular dynamics are recovered. The density profiles predicted without adjustable parameters are in a good agreement with the filtered densities from the simulations. Assuming spherical symmetry, we evaluate the height of the nucleation barrier and the Tolman length without adjustable parameters. The barrier heights calculated with the properties of the (111) and (110) interfaces envelope the Monte Carlo results, while those obtained with the average interface properties fall very close to the exact values. In contrast, the classical sharp interface model considerably underestimates the height of the nucleation barrier. We find that the Tolman length is positive for small clusters and decreases with increasing size, a trend consistent with computer simulations.
\end{abstract}

\section{INTRODUCTION}

In this paper we present a detailed quantitative test of the phase field theory of crystal nucleation in the hard sphere (HS) fluid, a case in which all the data necessary for such analysis are available.

Probably the least understood stage of the freezing of liquids is the initial nucleation phase in which the crystalline phase appears in the homogeneous liquid via heterophase fluctuations whose central part shows crystal-like atomic arrangement. Those heterophase fluctuations that exceed a critical size (determined by the interplay of the interfacial and volumetric contributions to the cluster free energy) have a good chance to reach macroscopic dimensions, while the smaller ones decay with a high probability. The description of such fluctuations is problematic even in single component systems. One of the main difficulties is that the typical size of the critical fluctuations that form on the human time scale $[1,2,3,4,5]$ is comparable to the thickness of the crystalliquid interface, which in turn extends to several molecular layers [6, 7, 8]. Therefore, the droplet model of the classical nucleation theory (CNT) that relies on a sharp interface and bulk crystal properties is expected to be inappropriate for describing such fluctuations. Field theoretic models, that predict a diffuse interface, offer a natural way to handle such problems [9]. For example, in a recent paper [10], the phase field theory (PFT) has been shown to describe such fluctuations quantitatively.

Due to extensive computer simulations done recently, the hard sphere (HS) liquid is probably the best known system that shows crystal nucleation. Its thermodynamic properties can be obtained accurately by integrating the equations of state of the crystalline and liquid phases evaluated from molecu- lar dynamics (MD) [11]. The interface density profiles are known for the (111) and (100) interfaces [8]. The free energy of the (111), (110), and (100) interfaces has been evaluated with a high accuracy [12]. Furthermore, the height of the nucleation barrier has been determined by Monte Carlo (MC) simulations [13]. This offers a unique possibility to test the abilities of various cluster models. For example, it has been established that the droplet model of the classical nucleation theory (that relies on the equilibrium value of the interface free energy) seriously underestimates the height of the nucleation barrier, and the agreement could only be restored via assuming that the interface free energy increases with supersaturation [5, 13]. Such behavior has been recovered by a single-order-parameter density functional approach based on a Ginzburg-Landau free energy consistent with face centered cubic (fcc) crystal symmetries [14]. Remarkably, however, the interface profiles from models deduced for fcc symmetries [14, 15, 16] do not fit well to the simulation results. It is, therefore, desirable to refine the field theoretic models so that details of the interface profiles are reproduced together with the height of the nucleation barrier. A possible starting point for such study is the phase field theory, that has been used successfully to describe many aspects of crystallization [17, 18], including nucleation [10].

Besides these, further interest in describing crystal nucleation in the hard sphere system is generated by recent experiments on colloidal suspensions that mimic closely the hardsphere behavior [19].

Herein, the hard sphere system is used to test the performance of the phase field theory. The model parameters of the phase field theory are fixed at the solid-liquid equilibrium so that the known interface thickness and free energy data are recovered. Then, the nucleation barrier height is predicted with- 
out adjustable parameters, which is compared to exact results from Monte Carlo simulations. It will be shown that the phase field theory leads to a considerable improvement relative to the classical droplet model. Having determined the interface density profiles, we evaluate the Tolman length, a quantity related to the curvature dependence of the interface free energy. We find that in agreement with computer simulations, the Tolman length is positive for small clusters and decreases towards a negative value with increasing cluster size.

\section{NUCLEATION THEORY}

\section{A. Phase field theory of nucleation}

In the present work, we apply two fields to describe the local state of matter: A non-conserved structural field $m$ called phase field, and the conserved volume fraction field $\phi$, which is related to the density field as $\phi=\frac{\pi}{6} \sigma^{3} \rho$, where $\sigma$ is the HS diameter, and $\rho$ is the number density. For historical reasons, $m$ is defined so that it is 1 for the liquid and 0 for the solid. Then, $1-m$ can be regarded as a local degree of crystallinity, and can be viewed as the Fourier amplitude of the dominant density wave in the Fourier expansion of the time averaged number density in the crystal.

In line with the formulation for binary systems $\lfloor 10,18,20$, 21], the Helmholtz free energy of the inhomogeneous hard sphere system is assumed to have the form

$$
F=\int d \mathbf{r}\left\{\frac{b T}{2}(\nabla m)^{2}+f(m, \phi)\right\},
$$

where the local Helmholtz free energy density is given as

$$
f(m, \phi)=w T g(m)+[1-p(m)] f_{S}(\phi)+p(m) f_{L}(\phi),
$$

the $g$ "double well" and $p$ "interpolation" functions have the usual form $g(m)=\frac{1}{4} m^{2}(1-m)^{2}$ and $p(m)=m^{3}(10-$ $\left.15 m+6 m^{2}\right)$, emerging from the thermodynamically consistent formulation of the phase field theory [20, 22], while $f_{S}(\phi)$ and $f_{L}(\phi)$ are the free energy densities of the bulk crystal and liquid phases. The square gradient (SG) term in the integrand is responsible for the diffuse interface. Being in unstable equilibrium, the critical fluctuation (the nucleus) can be found as an extremum of this free energy functional. Then the fields have to obey the appropriate Euler-Lagrange (EL) equations, which in the case of such local functional take the form

$$
\frac{\delta F}{\delta \chi}=\frac{\partial \psi}{\partial \chi}-\nabla \frac{\partial \psi}{\partial \nabla \chi}=0,
$$

where $\frac{\delta F}{\delta \chi}$ is the first functional derivative of the free energy with respect to the field $\chi(=m$ or $\phi)$, and $\psi$ is the total local free energy density [the integrand of Eq. (1)]. These equations have to be solved under the boundary conditions of having unperturbed liquid far from the fluctuation, and due to symmetry reasons, zero field gradients at the center of the fluctuations. Note, that since the volume fraction is a conserved field, we have to add $\lambda \int d \mathbf{r} \phi=-\mu_{\infty} \int d \mathbf{r} \rho$ to the right hand side of Eq. (1), when searching for the extremum of the free energy, where the Lagrange multiplicator $\lambda$ turns out to be proportional to the negative of the chemical potential $\mu_{\infty}$ of the unperturbed liquid. This is then the Legendre transformation that yields the grand potential, $\Omega=F-\mu_{\infty} N$, i.e., we seek the extremum of the grand potential functional. Assuming spherical symmetry, the EL equations boil down to the following ones

$$
\frac{\delta \Omega}{\delta m}=\frac{\partial \psi}{\partial m}-b T\left\{m^{\prime \prime}+\frac{2}{r} m^{\prime}\right\}=0
$$

and

$$
\frac{\delta \Omega}{\delta \phi}=\frac{\partial \psi}{\partial \phi}-\frac{\partial \psi}{\partial \phi}(r \rightarrow \infty)=0,
$$

where $r \rightarrow \infty$ means that the term has to be evaluated far from the fluctuation. [In this work' stands for differentiating with respect to the argument where argument is shown; otherwise (as above), it denotes differentiation with respect to the radial distance $r$ from the center of the fluctuation.] Note that Eq. (5) is an implicit equation for the volume fraction $\phi=\phi(m)$,

$$
0=[1-p(m)] \frac{\partial f_{S}}{\partial \phi}+p(m) \frac{\partial f_{L}}{\partial \phi}-\frac{\partial f_{L}}{\partial \phi}(r \rightarrow \infty)
$$

and thus

$$
\frac{\partial \psi}{\partial \phi}=w T g^{\prime}(m)+p^{\prime}(m)\left[f_{L}(\phi)-f_{S}(\phi)\right]
$$

is a function of $m$, i.e., Eq. (4) is an ordinary differential equation for $m$.

The properties of the critical fluctuation can be determined by solving Eq. (4) under the boundary conditions $m^{\prime} \rightarrow 0$ for $r \rightarrow 0$, and $m \rightarrow 1$ and $\phi \rightarrow \phi_{\infty}$ for $r \rightarrow \infty$ that correspond to the initial non-equilibrium liquid state. In this work, Eq. (4) has been solved numerically, using a variable 4th/5th order Runge-Kutta method. Since the boundary conditions fix $m$ and $m^{\prime}$ at different positions, the phase field value at the center, $m(r \rightarrow 0)$, for which the far-field condition is satisfied has been found iteratively.

Having determined $m(r)$, the volume fraction/density profile can be obtained by inserting it into the numerically inverted version of Eq. (6). The free energy of the critical fluctuation, in turn, can be calculated by inserting the solution $m(r)$ into

$$
W^{*}=\int_{0}^{\infty} d r 4 \pi r^{2} \Delta \omega[m, \phi(m)],
$$

where $\Delta \omega=\omega-\omega_{\infty}$, and the grand potential density is $\omega=$ $\psi-\mu_{\infty} \rho$, while $\omega_{\infty}=\psi_{\infty}-\mu_{\infty} \rho_{\infty}$. Here, subscript $\infty$ denotes properties of the initial liquid.

The interface free energy of small fluctuations is expected to depend on size or curvature due the reduction of the average number of solid neighbors of surface molecules relative to the planar interface. The analogous phenomenon in small liquid droplets has been studied extensively [23]. In the widely acknowledged thermodynamic theory of Tolman [24], the size 
dependence of the surface tension is given in terms of the Tolman length [25] $\delta_{T}=R_{e}-R_{p}$ (the distance of the equimolar surface [26] from the surface of tension [27]) as

$$
\gamma=\frac{\gamma_{\infty}}{1+2 \delta_{T} / R_{p}}
$$

where $\gamma_{\infty}$ is the surface tension for planar geometry. Although a rigorous derivation of these notions is unavailable for crystallites, a quantity analogous to $\delta_{T}$ has recently been evaluated from computer simulations [2]. It decreases with increasing size of the fluctuations. It is of considerable interest to see whether the phase field theory is able to reproduce this feature.

We determine the Tolman length here via three routes:

(i) Using the radius of the equimolar surface, $R_{e}=$ $\left\{\int_{0}^{\infty} d r 4 \pi r^{2}\left[\phi-\phi_{\infty}\right] /\left[(4 \pi / 3)\left(\phi_{0}-\phi_{\infty}\right)\right]\right\}^{1 / 3}$, where $\phi_{0}$ is the value at the center of the fluctuation, and the expression $R_{p}=\left[3 W^{*} /\left(2 \pi \Delta \omega_{\max }\right)\right]^{1 / 3}$ [28] for the radius of the surface of tension. Here $\Delta \omega_{\max }=\max \left\{f_{L}\left(\phi_{\infty}\right)+\right.$ $\left.\left(\partial f_{L} / \partial \phi\right)_{\phi_{\infty}}\left(\phi-\phi_{\infty}\right)-f_{S}(\phi)\right\}$ is the maximum driving force of crystallization, realized by the (compressed) solid that has the same chemical potential as the initial liquid [29].

(ii) On the basis of Eq. (9), $\delta_{T, \text { eff }}=\frac{1}{2} R_{p}\left(\gamma_{\infty} / \gamma-1\right)$, where the ratio of the curved and planar interfaces is related to the ration of the non-classical and classical cluster free energies as $\gamma / \gamma_{\infty}=\left(W^{*} / W_{C N T}^{*}\right)^{1 / 3}$ [28].

(iii) On the basis of Eq. (9), but approximating the radius of the surface of tension with that of the equimolar surface, $\delta_{T, \text { eff }}=\frac{1}{2} R_{e}\left(\gamma_{\infty} / \gamma-1\right)$, as done in the evaluation from MD results [2].

Note that Eq. (9) has been derived from the hypothesis of a size-independent Tolman length, a condition that is not satisfied in the known cases [30], thus routes (ii) and (iii) are expected to be less accurate.

Provided that $f_{L}(\phi)$ and $f_{S}(\phi)$ are known, the phase field theory contains only two parameters, the coefficient of the square-gradient term $b$ and the free energy scale $w$. It is worth recalling in this respect that these quantities can be related to the free energy and thickness of the equilibrium planar interfaces [29].

$$
\gamma_{\infty}=(2 b T)^{1 / 2} \int_{0}^{1} d \xi f[\xi, \phi(\xi)]^{1 / 2}
$$

and

$$
d_{10-90}=\left(\frac{b T}{2}\right)^{1 / 2} \int_{0.1}^{0.9} d \xi f[\xi, \phi(\xi)]^{-1 / 2},
$$

where $d_{10-90}$ is the $10 \%-90 \%$ thickness of the interface, the distance on which $m$ varies between 0.1 and 0.9 . Thus, if $\gamma_{\infty}$ and $d_{10-90}$ are known, these relationships can be used to fix $b$ and $w$ in equilibrium. This then allows the calculation of the free energy of the critical fluctuations $W^{*}$ and the Tolman length without adjustable parameters.

\section{B. Classical theory}

For comparison with our non-classical approach, we calculate the height of the nucleation barrier using the sharp in- terface droplet model of the classical nucleation theory [31]. In this approach, the free energy of heterophase fluctuations of radius $R$ is given as $W_{C N T}=-(4 \pi / 3) R^{3} \Delta \omega_{\max }+$ $4 \pi R^{2} \gamma_{\infty}$. Then, the free energy of the critical fluctuation of radius $R_{C N T}^{*}=2 \gamma_{\infty} / \Delta \omega_{\max }$ reads as $W_{\mathrm{CNT}}^{*}=$ $(16 \pi / 3) \gamma_{\infty}^{3} \Delta \omega_{\max }^{-2}$

\section{Application to hard spheres}

The stable crystalline phase of the hard sphere system has the face centered cubic (fcc) structure. The nuclei, in turn, contain fcc (ABC) and hexagonal close packed (hcp) stacking (ABA) with equal probability [5], indicating that the nucleation barrier for them is rather similar. Therefore, we address here only fcc nucleation.

The Gibbs free energy difference between the fcc solid and liquid (the driving force of crystallization) has been obtained by integrating the virtually accurate equations of state by Hall [11] (that fit to the molecular dynamics results), starting from the volume fractions of the coexisting solid and liquid $\phi_{\mathrm{fcc}, \mathrm{coex}}=0.546$ and $\phi_{\mathrm{L}, \mathrm{coex}}=0.494$ at pressure $p=11.81 \mathrm{kT} / \sigma^{3}$. Polynomials were then fitted to the chemical potentials of the solid and liquid phases, whose relative deviation from the accurate chemical potentials are less then $10^{-4}$ in the density range of interest. These polynomials were then integrated analytically to obtain the free energy densities of the two bulk phases.

Since the structural order parameter $1-m$ can be interpreted as the Fourier amplitude of the dominant density waves (with the first neighbor reciprocal lattice vectors as the wave number), we identify the phase field profile as the crossinterfacial variation of the height of the density peaks. This is an approximation, since other Fourier components also contribute. However, as pointed out by Shen and Oxtoby [15], so far as the density of solid is reasonably approximated by Gaussians centered around the fcc lattice sites, the amplitudes of all other components are proportional to the amplitude of the dominant wave, i.e., to a first approximation the height of the density peaks is proportional to the amplitude of the dominant Fourier component.

The interfacial density profiles of the equilibrium hardsphere crystal-liquid interfaces of orientations (100) and (111) have been studied by Davidchack and Laird using molecular dynamics simulations [8]. We have received new high resolution data from them for the (111), (100), and (110) interfaces. In the latter cases, the bin size for the density profile was $1 / 32$ times the layer width, while the parameters for Gaussian filtering were $N=64$ and $\epsilon=28.1$ [32]. (For definitions see [8].) The new density distributions are fully consistent with those in [8]. From the interfacial variation of the peak amplitudes, we obtained $5.94 \sigma, 4.75 \sigma$, and $5.54 \sigma$ for the $10 \%-90 \%$ interface thickness for the (111), (110), and (100) directions, respectively.

The same authors evaluated the free energy of the fcc crystal - liquid interface for these orientations [12]: $\gamma_{\infty} \sigma^{2} / k T=$ $0.58 \pm 0.01,0.62 \pm 0.01$, and $0.64 \pm 0.01$ for the (111), (100) and (110) interfaces. Considering that due to the 


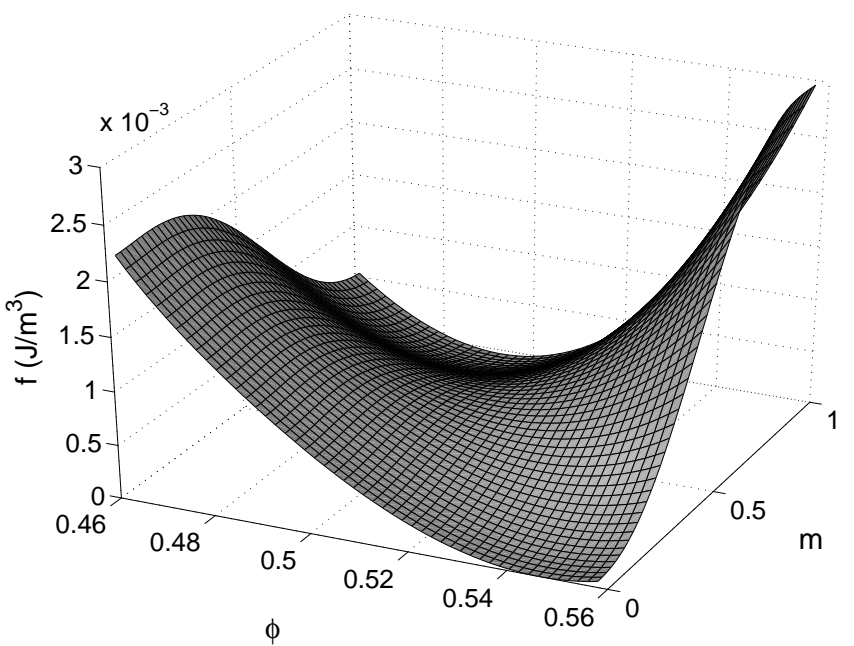

FIG. 1: Free energy surface for the hard sphere system evaluated with free energy scale $w$ corresponding to the (111) interface. Note, that the minima at $(\phi=0.494, m=1)$ and $(\phi=0.546, m=$ 0 ) correspond to the equilibrium liquid and solid (fcc) phases. The calculation has been performed for a colloidal suspension of hard sphere diameter $\sigma=890 \mathrm{~nm}$, a particle size comparable to that in many experiments [19]. The small value of the free energy density follows from this large molecular diameter.

small anisotropy the equilibrium shape is expected to be nearly spherical, we also performed calculations with an average of the interface free energy over the known orientations $\gamma_{\infty} \sigma^{2} / k T=0.6133$

Having this information available, the model parameters $b$ and $w$ were determined using Eqs. (10) and (11) via Newton-Raphson iteration. The Helmholtz free energy density [Eq. (2)] obtained with $w$ evaluated using the properties of the (111) interface is shown in Fig. 1. Qualitatively similar free energy surfaces were obtained for the other orientations.

Using these data, the PFT and CNT predictions for the nucleation barrier can be made free of adjustable parameters.

\section{RESULTS AND DISCUSSION}

\section{A. Density profiles}

Fixing the model parameters $b$ and $w$ as described above, the phase field profile for the equilibrium solid-liquid interface can be determined via the equation [29]

$$
z(m)=\left(\frac{b T}{2}\right)^{1 / 2} \int_{0.5}^{m} d \xi f[\xi, \phi(\xi)]^{-1 / 2},
$$

where $z$ is the distance perpendicular to the plane $m=0.5$. The coarse grained density profile that corresponds to the filtered density by Davidchack and Laird [8] can be obtained by inserting the solution $m(z)$ into Eq. (6) after inverting it numerically.

The predicted structural order parameter $(1-m)$ and normalized coarse grained density $\left(\rho-\rho_{\min }\right) /\left(\rho_{\max }-\rho_{\min }\right)=$

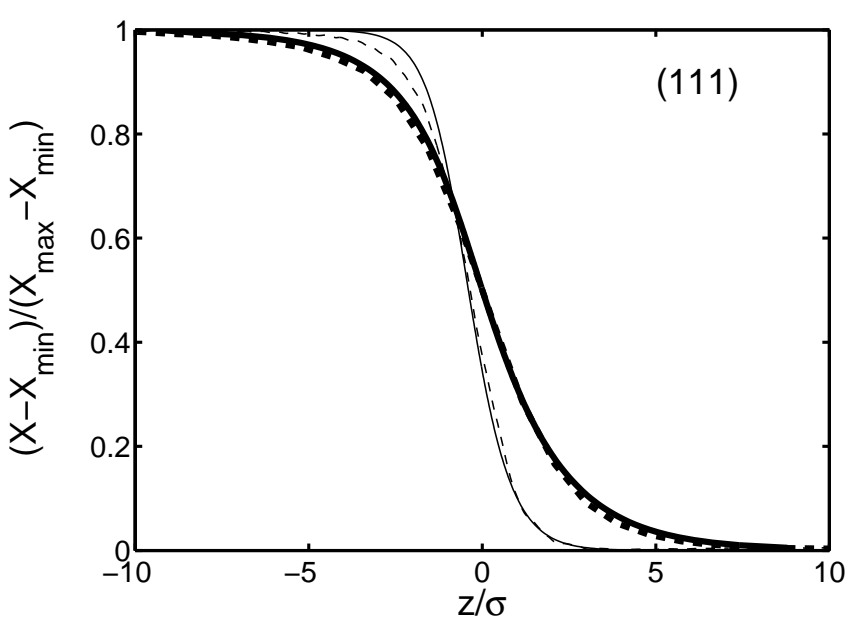

FIG. 2: Reduced interfacial profiles for the (111) interface. (Solid lines: predictions of the phase field theory; heavy solid line - structural order parameter, $1-m$; light solid line - density. Dashed lines: Results from molecular dynamics [8]; heavy dashed line - height of singlet density peaks; light dashed line - filtered density profile. Note that the heavy lines should be compared to each other, as the light ones.)

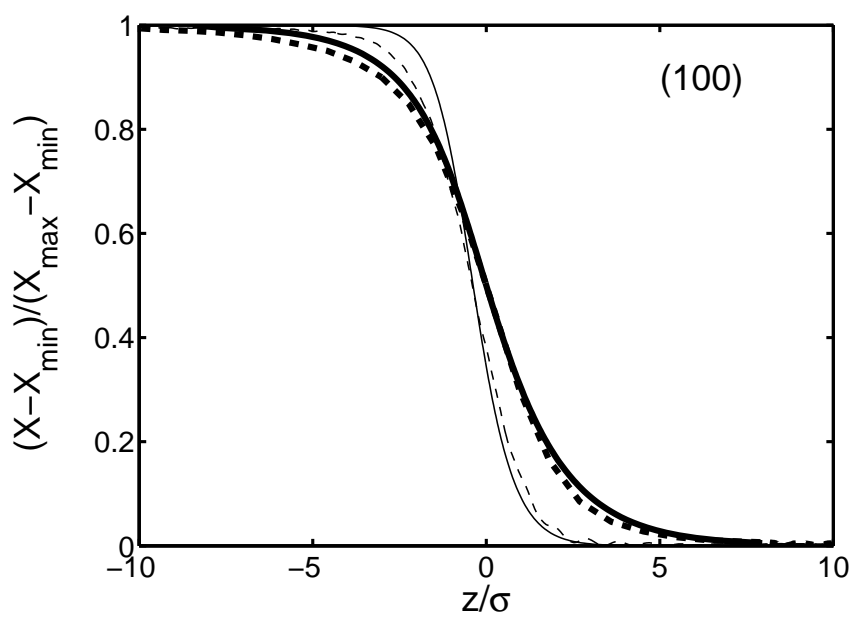

FIG. 3: Reduced interfacial profiles for the (100) interface. Notation as for Fig. 2 .

$\left(\phi-\phi_{\min }\right) /\left(\phi_{\max }-\phi_{\min }\right)$ profiles are compared with their counterparts from molecular dynamics simulations in Figs. 2 to 4 . For the sake of comparison, the simulation results are also presented in a normalized form, $\left(X-X_{\min }\right) /\left(X_{\max }-\right.$ $\left.X_{\min }\right)$, where $X$ is either the density peak height or the filtered density, while $X_{\min }$ and $X_{\max }$ are the minimum and maximum values of these quantities.

For the (111) and (100) interfaces, and to a smaller extent for the (110) interface, the structural order parameter profiles are in a good agreement with the cross-interfacial variation of the density peak height from MD. Note that while $d_{10-90}$ has been fitted, the agreement depends also on the shape/symmetry of the predicted profile. It appears, that the nearly symmetric structural order parameter profile of the PFT 


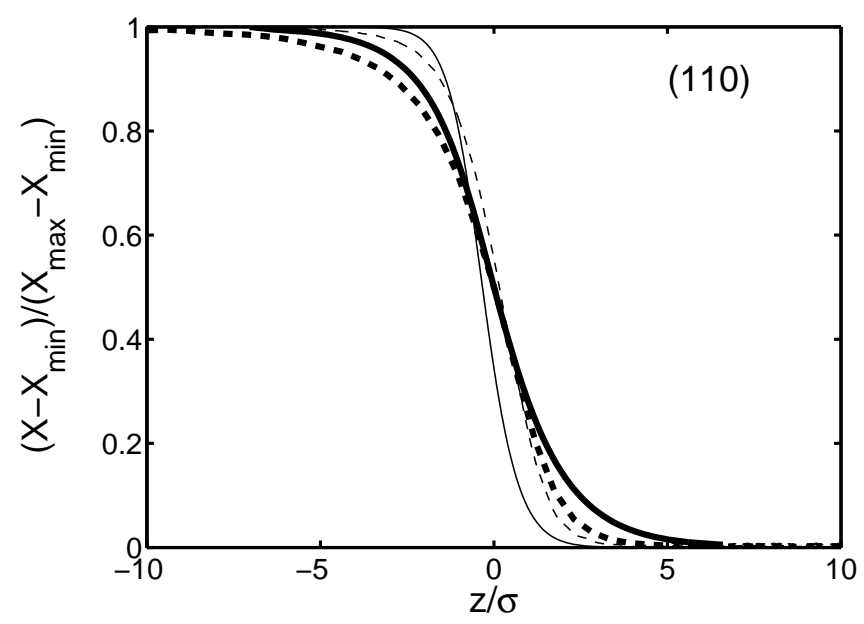

FIG. 4: Reduced interfacial profiles for the (110) interface. Notation as for Fig. 2.

approximates reasonably well the behavior seen in the simulations. Remarkably, the density functional theories by Shen and Oxtoby [15, 16], and Gránásy and Pusztai [14] derived for the fcc structure predict significantly more asymmetric structural order parameter profiles for the crystal-liquid interfaces, which are sharper on the crystal side and have a long tail on the liquid side. Therefore, their match to the simulated profiles is apparently less satisfactory. It is an intriguing question why the quartic form of $g(m)$, that is consistent with the symmetries of the base centered cubic (bcc) structure [33], leads to a better fit to the simulation profile then those obtained by using free energy functionals consistent with the fcc symmetries. A distinct possibility is the presence of a bcc-like layer at the interface as reported in the case of the Lennard-Jones system [4, 15]. This question, however, could only be addressed in a full density functional theory (such as that by Shen and Oxtoby [34]) that incorporates the possibility for the Bain distortion that connects the bcc and fcc structures.

The coarse grained density profiles can now be predicted in the PFT without adjustable parameters. They appear to be in a good agreement with the simulation results for the (111) and (100) interfaces (Figs. 2 and 3), however, a shift of about half a $\sigma$ can be seen between them for the (110) direction, the PFT result lying closer to the crystal (Fig. 4).

Summarizing, in the PFT, we have a free energy surface and a SG coefficient that reproduce exactly the thermodynamics of the bulk phases, the interface free energy, and quite reasonably both the structural order parameter profile and the density profile. Thus, we may attempt to calculate the height of the nucleation barrier with some confidence.

\section{B. Nucleation}

The reduced nucleation barrier heights calculated with $b$ and $w$ that fit to the properties of the (111) and (110) interfaces and to the average interface properties are shown in Fig. 5 for the phase field theory. For comparison, the predictions of the

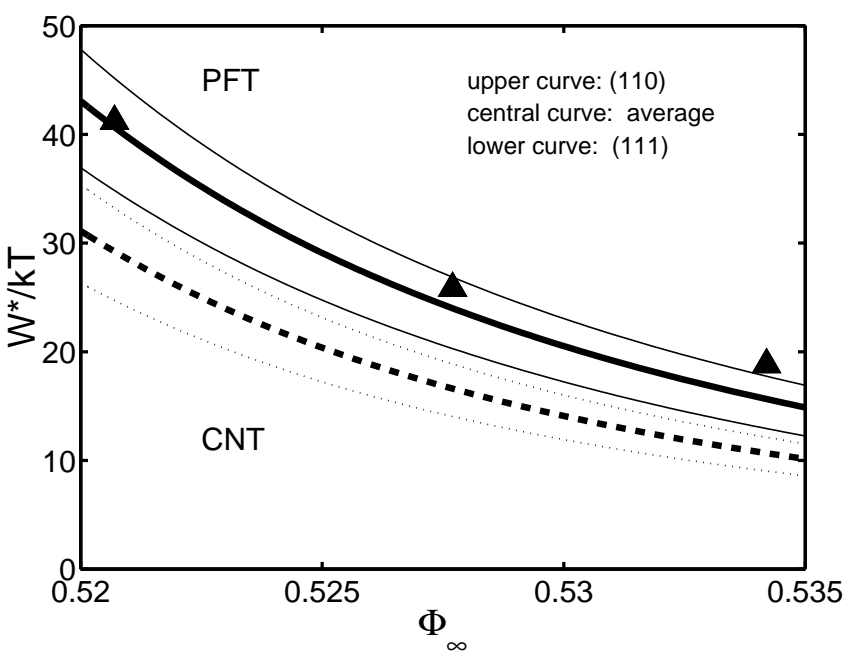

FIG. 5: Reduced nucleation barrier height vs. volume fraction of the initial liquid. (Solid lines: phase field theory - PFT; dashed/dotted lines: classical droplet model - CNT.) The upper an lower curves were calculated using the physical properties of the (111) and (110) interfaces, respectively. The prediction for the (100) interface (not shown here) falls slightly below the results obtained with the average interface properties (heavy lines). For comparison, the results of Monte Carlo simulations [13] are also shown (triangles).

classical nucleation theory and the exact results from Monte Carlo simulations [13] are also presented. The $W^{*}$ vs. initial liquid volume fraction curves predicted by the PFT using the properties of the (111) and (110) interfaces envelope the MC simulations, while the predictions with the average interface properties fall close to the MC results. The slope of the $W^{*}$ vs. $\phi_{\infty}$ curve appears to be somewhat larger for the PFT predictions than for the MC simulations. A possible explanation could be the density dependence of the parameters $b$ and $w$ [14]. However, the more complex Euler-Lagrange equations associated with such problem will be investigated elsewhere.

It is of interest to determine the magnitude of the error associated with these results. While the relevant thermodynamic data are virtually exact, uncertainties of $\left( \pm 0.01 k T / \sigma^{2}\right)$ and $( \pm 5 \%)$ are associated with the interface free energy and the interface thickness. We find that for $\phi=0.5207$ the barrier height is $W^{*} / k T=40.63$, while its uncertainties originating from those of the interface free energy and the interface thickness amount to \pm 1.91 and \pm 0.23 , respectively, yielding \pm 1.92 when assuming Gaussian error propagation. The magnitude of these errors varies approximately proportionally with the barrier height. These errors do not influence the validity of our conclusions.

The radial order parameter and density profiles corresponding to the volume fractions used in MC simulations $(0.5207$, 0.5277 , and 0.5343) are shown in Fig. 6. The interfacial profiles for $\phi_{\infty}=0.5207$ are in a reasonable agreement with the size of the respective critical fluctuation from MC simulation (cf. our Fig. 6 and Fig. 3 of Ref. [5]). Note that the bulk crystalline properties have not been established even at the centers of the critical fluctuations. Accordingly, the clas- 


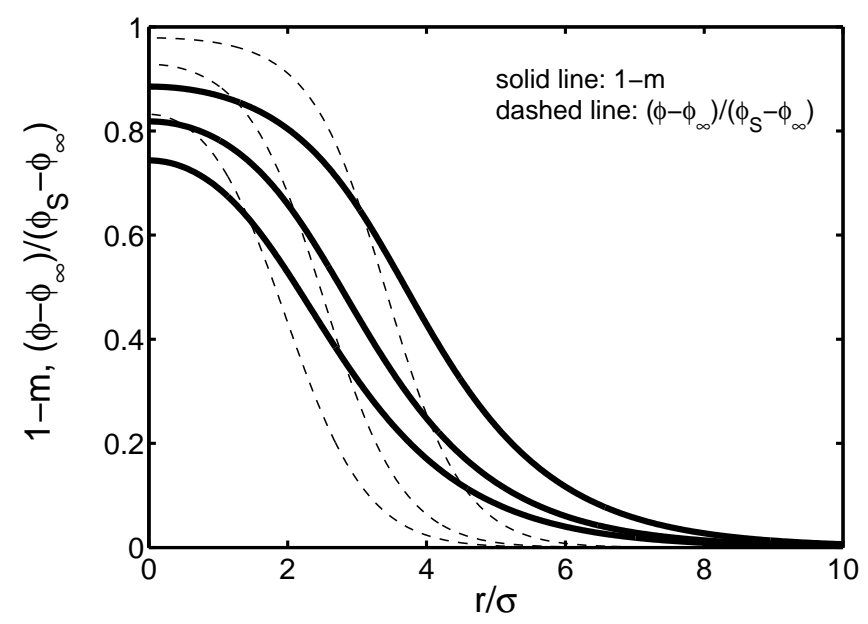

FIG. 6: Radial structural order parameter (solid lines) profiles and reduced density (dashed lines) profiles at initial liquid volume fractions $\phi_{\infty}=0.5207,0.5277$, and 0.5343 , calculated with $b$ and $w$ corresponding to the average interface properties $\left(\gamma_{\infty} \sigma^{2} / k T=0.6133\right.$ and $\left.d_{10-90} / \sigma=5.410\right)$. Here $\phi_{S}(=0.5796,0.5875$, and 0.5948 , respectively) is the volume fraction of the solid that is in mechanical equilibrium with the initial liquid (the crystal that has the same pressure as the liquid of volume fraction $\phi_{\infty}$ ).

sical droplet model significantly underestimates the height of the nucleation barrier for all orientations. Since the nucleation rate is proportional to $\exp \left(-W^{*} / k T\right)$, these differences amount to orders of magnitude. For example, the calculation made with the average interface free energy overestimates the nucleation rate by three to five orders of magnitude. At the volume fraction $\phi_{\infty}=0.5781$, the PFT and CNT predictions intersect each other. In both approaches, the height of the nucleation barrier decreases monotonically with the supersaturation.

The three known orientations can in principle be used to approximate the anisotropy of the interface free energy 35 , 36], that in turn defines the equilibrium shape of minimum interface free energy for given volume. Since the nuclei are expected to minimize their free energy, their average shape can probably be reasonably approximated by the equilibrium form. Work is underway to determine the nucleation barrier in such case.

Finally, we draw attention to the fact, that the MC data for $W^{*} / k T$ [5, 13], used here as reference, were obtained directly via "umbrella sampling", therefore, possible uncertainties associated with the nucleation prefactor [3] do not plague them.

\section{Tolman length}

The Tolman lengths calculated for the critical fluctuations along the three routes specified above are shown as a function of the initial volume fraction in Fig. 7. Similarly to the field theoretic results for vapor-liquid nucleation [30], for small sizes the Tolman length is positive and comparable to the molecular diameter, while $\delta_{T}$ decreases with increasing

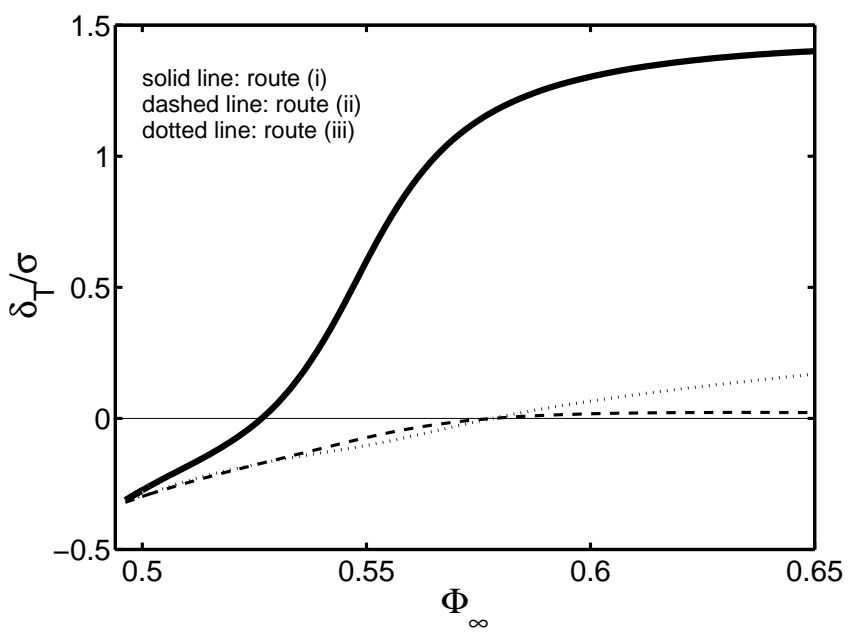

FIG. 7: Tolman length as a function of initial volume fraction calculated along routes (i)-(iii).

size towards a negative limiting value for the planar interface. Although this behavior is general for the Tolman lengths from all three routes, routes (ii) and (iii) yield considerably smaller values than the more accurate route (i). Accordingly, the change of sign happens at different volume fractions: At $\phi_{\infty}=0.5266$ for route (i), while at $\phi_{\infty}=0.5781$ for routes (ii) and (iii). The differences originate from the fact that the assumption $\delta_{T}=$ const., routes (ii) and (iii) rely on, is not satisfied for small clusters. In turn, in the large particle limit this assumption becomes valid. Indeed, the Tolman lengths from all three routes converge to the same negative value $\left(\delta_{T, e q} / \sigma=-0.33\right)$ in the planar limit.

\section{SUMMARY}

We presented a phase field theory of homogeneous crystal nucleation in hard sphere liquid, that describes local state of matter via a structural order parameter field and the density field.

A rigorous test of the model is performed using the virtually exact thermodynamic properties of the bulk phases. The two model parameters are chosen so that the free energy and the $10 \%-90 \%$ thickness for the cross-interfacial variation of the density peak height known from molecular dynamics simulations are recovered. For the (111) and (100) interfaces, and to a lesser extent for the (110) interface, the resulting structural order parameter and density profiles are in a significantly better agreement with the computer simulations, than seen for previous continuum models. Without adjustable parameters, the phase field theory predicts the height of the nucleation barrier with a reasonable accuracy. This represents a considerable improvement over the sharp interface droplet model of the classical nucleation theory, which is known to overestimate the nucleation rate by several orders of magnitude.

The cross-interfacial density profiles are used to calculate the Tolman length for critical fluctuations, which is positive 
for small sizes, and tends to a negative value in the large cluster limit.

\section{Acknowledgments}

We thank R. Davidchack and B. B. Laird for com- municating us their new high resolution density data for the (111), (110), and (100) interfaces prior to publication and for the illuminating discussions. This work has been supported by the Norwegian Research Council under project Nos. 153213/432 and 151400/210, by the ESA under Prodex Contract No. 14613/00/NL/SFe, and by the Hungarian Academy of Sciences under contract No. OTKA-T-037323.
[1] W. C. Swope and H. C. Andersen, Phys. Rev. B 41, 7024 (1990).

[2] L. A. Báez and P. Clancy, J. Chem. Phys. 102, 8138 (1995).

[3] L. S. Bartell, Annu. Rev. Phys. Chem. 49, 43 (1998).

[4] P. R. ten Wolde, M. J. Ruiz-Montero, and D. Frenkel, Phys. Rev. Lett. 75, 2714 (1995); J. Chem. Phys. 104, 9932 (1996).

[5] S. Auer and D. Frenkel, Nature 409, 1020 (2001).

[6] J. Q. Broughton and H. H. Gilmer, J. Chem. Phys. 84, 5759 (1986).

[7] B. B. Laird and A. D. J. Haymet, Chem. Rev. 92, 1819 (1992).

[8] R. L. Davidchack and B. B. Laird, J. Chem. Phys. 108, 9452 (1998).

[9] D. W. Oxtoby, Annu. Rev. Mater. Res. 32, 39 (2002).

[10] L. Gránásy, T. Börzsönyi, and T. Pusztai, Phys. Rev. Lett. 88, art. no. 206105 (2002).

[11] K. R. Hall, J. Chem. Phys. 57, 2252 (1970).

[12] R. L. Davidchack and B. B. Laird, Phys. Rev. Lett. 85, 4751 (2000).

[13] S. Auer and D. Frenkel, Nature 413, 711 (2001).

[14] L. Gránásy and T. Pusztai, J. Chem. Phys. 117, 10121 (2002).

[15] Y. C. Shen and D. W. Oxtoby, J. Chem. Phys. 104, 4233 (1996).

[16] Y. C. Shen and D. W. Oxtoby, J. Chem. Phys. 105, 6517 (1996).

[17] W. J. Boettinger, J. A. Warren, C. Beckermann, A. Karma, Annu. Rev. Mater. Res. 32, 163 (2002).

[18] L. Gránásy, T. Pusztai, J. A. Warren, J. A. Douglas, T. Börzsönyi, and V. Ferreiro, Nature Materials 2, 92 (2003).

[19] D. Rosenbaum, P. C. Zamora, and C. F. Zukoski, Phys. Rev. Lett. 76, 150 (1996); J. L. Harland and W. van Mengen, Phys. Rev. E 55, 3054 (1997); P. Bartlett and P. B. Warren, Phys. Rev. Lett. 82, 1979 (1999); Review: T. Palberg, J. Phys.: Condens. Matter 11, R323 (1999); U. Gasser, E. R. Weeks, A. Schofield, P. N. Pusey, and D. A. Weitz, Science 292, 258 (2001).

[20] J. A. Warren and W. J. Boettinger, Acta Metall. Mater. 43, 689 (1995).

[21] G. Caginalp and J. Jones, Ann. Phys. 237, 66 (1995).

[22] S. L. Wang, R. F. Sekerka, A. A. Wheeler, B. T. Murray, S. R. Corriell, R. J. Braun, G. B. McFadden, Physica D 69, 189 (1993).

[23] J. S. Rowlinson and B. Widom, Molecular Theory of Capillarity (Claredon, Oxford, 1982).
[24] R. C. Tolman, J. Chem. Phys. 17, 333 (1949).

[25] Tolman's original definition. A different definition is applied by several authors: $\delta_{T}=\lim _{R \rightarrow \infty} R_{e}-R_{p}$. In our terminology, the latter corresponds to the equilibrium Tolman length, $\delta_{T, e q .}$.

[26] Defined by the position of a step-function that has the same spatial integral and amplitude as the interfacial density profile.

[27] The surface at which the surface tension acts. It is the surface for which the generalized Laplace equation, $\Delta p_{c}=2 \gamma / R+$ $\partial \gamma / \partial R$ reduces to the original one; i.e., $\partial \gamma / \partial R=0$. Here $\Delta p_{c}$ is the capillary pressure.

[28] The radius of the surface of tension can be expressed as $R_{p}=$ $\left[3 W^{*} /\left(2 \pi \Delta \omega_{\max }\right)\right]^{1 / 3}$, while the interface free energy acting at the radius of the surface of tension is defined as $\gamma=$ $\left[\left(3 W^{*} \Delta \omega_{\max }^{2}\right) / 16 \pi\right]^{1 / 3}$ [see e.g., K. Koga, X. C. Zeng, and A. X. Shcheckin, J. Chem. Phys. 109, 4063 (1998) or L. Gránásy, ibid. 1045188 (1996)]. Then, $\gamma / \gamma_{\infty}=\left(W^{*} / W_{C N T}^{*}\right)^{1 / 3}$.

[29] J. W. Cahn and J. E. Hilliard, J. Chem. Phys. 28, 258 (1958); ibid. 31, 688 (1959).

[30] Field theoretic models of the vapor-liquid interface predict a strongly size dependent Tolman length that has a positive value for small droplets and tend to a negative value in the large particle limit. See e.g.: M. P. A. Fisher and M. Wortis, Phys. Rev. B 29625 (1984); M. Iwamatsu, J. Phys.: Condens. Matter 6, L173 (1994); I. Hadjiagapiou, ibid. 6, 5303 (1994); V. Talanquer and D. W. Oxtoby, J. Phys. Chem. 99, 2865 (1995); A. E. van Giessen, E. M. Blokhuis, and D. J. Bukman, J. Chem. Phys. 108, 1148 (1998); K. Koga, X. C. Zeng, and A. X. Shcheckin, ibid. 109, 4063 (1998); L. Gránásy, ibid. 109, 9660 (1998); J. Barrett, ibid. 111, 5938 (1999).

[31] See for example K. F. Kelton, Solid State Phys. 45, 75 (1991).

[32] R. Davidchack, personal communication.

[33] W. H. Shih, Z. Q. Wang, X. C. Zeng, and D. Stroud, Phys. Rev. A 35, 2611 (1987).

[34] Y. C. Shen and D. W. Oxtoby, J. Phys.: Condens. Matter 8, 9657 (1996); Phys. Rev. Lett. 77, 3585 (1996).

[35] R. Davidchack and B. B. Laird, J. Chem. Phys. 118, 7651 (2003).

[36] J. J. Hoyt, M. Asta, and A. Karma, Phys. Rev. Lett. 86, 5530 (2001). 